\title{
Am individuellen Therapieergebnis orientierte Erstattungsverfahren in der Onkologie: ethische Implikationen am Beispiel der CAR-T-Zelltherapie
}

\author{
Julia König • Christoph Gerst • Lorenz Trümper • Gerald G. Wulf • \\ Claudia Wiesemann
}

Online publiziert: 13. Februar 2020

(C) Der/die Autor(en) 2020

Im März 2019 meldete die Novartis Pharma GmbH, dass sie sich mit der GWQ ServicePlus (Gesellschaft für Wirtschaftlichkeit und Qualität bei Krankenkassen) auf ein Erstattungsverfahren zur Finanzierung der CAR-T-Zelltherapie mit Tisagenlecleucel geeinigt habe (Madlberger 2019). Das neue Erstattungsverfahren sieht eine am individuellen Therapieergebnis orientierte Erstattung vor. Die Jahrestherapiekosten/Patient mit Tisagenlecleucel betragen 320.000,00€ (Gemeinsamer Bundesausschuss 2019). Die Novartis Pharma GmbH erstatte allerdings einen Teil der Kosten, wenn die oder der damit behandelte Patientin oder Patient einen bestimmten Zeitraum nicht überlebt (Madlberger 2019).

Bei der CAR-T-Zellentherapie handelt es sich um eine zelluläre Immuntherapie, die bei Patienten mit refraktärer oder rezidivierter akuter lymphatischer B-ZellLeukämie (ALL) und bei Patienten mit rezidiviertem oder refraktärem diffusen großzelligen B-Zell-Lymphom (DLBCL) eingesetzt wird (Madlberger 2019; Bach et al. 2017; European Medicines Agency 2018). Hierfür werden körpereigene T-Lymphozyten gewonnen und ex vivo genetisch modifiziert, um nach Reinfusion in vivo das Immunsystem gegen die Leukämie- oder Lymphomzellen zu richten (Bach et al.

Die Autoren G.G. Wulf und C. Wiesemann teilen sich die Letztautorenschaft.

Dr. med. J. König, MAE ( $\varangle)$ · Prof. Dr. med. L. Trümper · Prof. Dr. med. G. G. Wulf Klinik für Hämatologie und medizinische Onkologie, Universitätsmedizin Göttingen, Robert-Koch-Str. 40, 37075 Göttingen, Deutschland

E-Mail: julia.koenig@med.uni-goettingen.de

Dr. phil. C. Gerst

Medizinrecht und Forschungsrecht, Stabstelle Recht, Universitätsmedizin Göttingen, Robert-Koch-Str. 40, 37075 Göttingen, Deutschland

Prof. Dr. C. Wiesemann

Institut für Ethik und Geschichte der Medizin, Universitätsmedizin Göttingen, Humboldtallee 36, 37073 Göttingen, Deutschland 
2017). Das Hauptziel der Therapie mit CAR-T Zellen bei Patienten mit Leukämien und Lymphomen ist die Verlängerung menschlichen Lebens. Dieses Ziel wurde in den pivotalen klinischen Prüfungen der aktuell zugelassenen Präparate gemessen an den Gesamtüberlebenszeiten als primären Endpunkten der jeweiligen Studien erreicht (Lin et al. 2019; Neelapu et al. 2018).

Tisagenlecleucel ist zugelassen als Arzneimittel zur Behandlung eines seltenen Leidens (Orphan medicinal product, Orphan Drug) nach der Verordnung (EG) Nr. 141/2000 des Europäischen Parlaments und des Rates vom 16. Dezember 1999 über Arzneimittel für seltene Leiden sowie als Arzneimittel für neuartige Therapien (ATMP) nach der Verordnung 1394/2007 des Europäischen Parlaments und des Rates vom 13. November 2004 und gilt wohl auch als „Blutzubereitung“ im Sinne von $\S 4$ Abs. (2) AMG (Arzneimittelgesetz), weil für seine Herstellung die köpereigenen T-Zellen der Patienten benötigt werden (European Medicines Agency 2018). Orphan Drugs, ATMPs und Blutzubereitungen sind definitionsgemäß als Arzneimittel nach $\S 2$ AMG einzustufen. Damit hätte die Preisgestaltung des Produkts nach den Vorgaben des Arzneimittelrechts zu erfolgen. Doch da die CAR-T-Zellen-Produkte nicht als Fertigarzneimittel nach $\S 4$ Nr. 1 AMG anzusehen sind, gilt die diesbezüglich nach § 78 AMG vorgeschriebene Arzneimittelpreisverordnung nicht. Damit sind den Herstellern bei der Preisgestaltung von CAR-T-Zellenprodukten gesetzlich zurzeit keine Schranken auferlegt.

Einen am individuellen Therapieergebnis orientierten Preisanteil eines Medikaments festzulegen ist bislang im deutschen Gesundheitssystem nicht üblich. Mit seiner Einführung wird nicht nur das bestehende Vertrags- und Abrechnungskonzept verändert, sondern auch das Konzept eines am Gruppennutzen ermittelten, in der zugelassenen Therapiesituation bestehenden a-priori Preises durch das eines a-posteriori, am konkreten Nutzen für ein Individuum ermittelten Preises ersetzt.

Ein solches Erstattungsverfahren wirft ethische Fragen auf, die geprüft werden sollten, bevor das Verfahren zum Einsatz kommt. In einem am individuellen Therapieergebnis orientierten Erstattungsverfahren müssen für diese Therapien nur dann die Gesamtkosten gezahlt werden, wenn ein menschliches Leben über einen Grenzwert hinaus verlängert wird. Bei kürzerer Überlebenszeit wird die Hälfte der Kosten zurückerstattet. Damit bewertet der am Therapieergebnis orientierte Preis für eine CAR-T-Zelltherapie direkt a-posteriori eine Zeitspanne individuellen menschlichen Lebens. Da dem Gut des menschlichen Lebens in der Ethik ein moralischer Status zugeschrieben wird (Beauchamp und Childress 2013, S. $62 \mathrm{ff}$.), wird mit einer monetären Bewertung menschlichen Lebens die deskriptive Ebene verlassen und eine normative Position eingenommen. Das am individuellen Therapieergebnis orientierte Erstattungsverfahren impliziert also eine normative Bewertung menschlichen Lebens und muss sich daher mit ethischen Maßstäben messen lassen. Dies muss vor dem Horizont der im jeweiligen Gesundheitssystem geltenden ethischen Grundüberzeugungen geschehen. In Deutschland ist dies die Orientierung an der Menschenwürde auf der einen Seite, sowie das Prinzip der Chancengleichheit beim Zugang zu Gesundheitsleistungen auf der anderen Seite (Deutscher Ethikrat 2011, S. 12, 73). 


\section{Begriffliche Klärung}

Zur Beschreibung des hier erörterten Erstattungssystems verwenden die pharmazeutischen Firmen den Begriff ,pay for performance“. Unter dieser Bezeichnung wird es derzeit auch in der deutschen Literatur diskutiert (Korzilius et al. 2019; Burgdorf et al. 2009). „Pay for performance“, auch P4P abgekürzt, zu Deutsch: erfolgsabhängige Erstattung, bezeichnet im angloamerikanischen Raum Programme, die die Höhe des ärztlichen Gehalts an den globalen Erfolg bei der Behandlung einer bestimmten Erkrankung oder bei der Früherkennung koppeln. Um die Qualität der ärztlichen Versorgung zu verbessern, wird so für den Arzt ein Anreiz geschaffen, in seiner Praxis etablierte Programme einzusetzen, die nachgewiesenermaßen für die Gruppe der Patienten einen Mehrwert darstellen (Lin et al. 2019; Matthes 2019). Die Bezeichnung ist jedoch für das hier verhandelte Erstattungsmodell irreführend. Bei der CAR-T-Zelltherapie wird zum einen nicht die Qualität der ärztlichen Behandlung gemessen an dem für eine Gruppe von Patienten erzielten Nutzen bewertet. Zum anderen geht es nicht darum, einen Anreiz für eine bessere Versorgung zu schaffen, da der individuelle Erfolg der CAR-T-Zelltherapie bis dato nicht vorhersagbar ist. Um diese Unterschiede deutlich zu machen, plädieren wir dafür, von einem am individuellen Therapieergebnis orientierten Erstattungsverfahren (,,pay for individual outcome“, kurz: P4IO) zu sprechen.

\section{Welche ethischen Prämissen liegen der Bewertung menschlichen Lebens im Rahmen der Preisfindung zugrunde?}

Die normative Bewertung des P4IO-Verfahrens besteht darin, das Überleben eines Menschen zum Maßstab für Therapieerfolg zu machen und dafür wiederum einen Preis anzusetzen. Dies entspricht einer Bewertung menschlichen Lebens nach dem naturalistischen Standard. Der Begriff ,naturalistischer Standard“ entstammt der Diskussion über die angemessene Bewertung von Lebensqualität (Quante 2014, S. 33 ff.). Eine Bewertung des menschlichen Lebens und seiner Qualität nach dem naturalistischen Standard basiert ausschließlich auf biologischen und medizinischen Fakten, wie hier etwa der reinen Überlebenszeit. Leben und Lebensqualität kann jedoch nach Quante normativ nur auf einem personalen oder einem intersubjektivrationalen Standard bewertet werden. Der Wert menschlichen Lebens sollte vor dem Hintergrund der Menschenwürde und auf der Basis des personalen Standards mit Rückgriff auf die Wertvorstellungen der jeweilig betroffenen Person ermittelt werden und damit ihrer autonomen Entscheidung unterliegen. Dabei muss berücksichtigt werden, dass sich der Mensch als Person in einem kontinuierlichen evaluativen Prozess befindet, in dem er sich und seine Interessen reflektiert (Quante 2014, S. 33 ff.). Eine Bewertung menschlichen Lebens durch andere Akteure als durch die betroffene autonome Person ist nach Quante nicht zulässig. Ersatzweise kann bei nicht autonomen Personen auf den intersubjektiv-rationalen Standard ausgewichen werden, welcher dann auf Merkmalen beruht, die rationale Subjekte als Ergebnis einer intersubjektiven Verständigung vernünftigerweise wählen würden. Dazu zählen Befriedigung sozialer Bedürfnisse, Empfindungsvermögen etc., nicht aber das reine 
Überleben. Somit sollte der Wert des menschlichen Lebens nicht allein an der Fähigkeit zu überleben gemessen werden und insbesondere nicht allein durch wenige, ausgewählte Dritte festgelegt werden. Ein solches Werturteil auf der Basis monetärer Erstattung, wie es im Rahmen des am individuellen Therapieergebnis orientierten Erstattungsverfahrens erfolgt, ist damit nicht statthaft.

Zudem muss gefragt werden, ob die monetäre Bewertung des individuellen Therapieergebnisses, gemessen am Überleben, mit dem Prinzip der Chancengleichheit vereinbar ist. Chancengleichheit wird in Frage gestellt, wenn die Bewertung menschlichen Lebens nicht von allen Betroffenen mitgetragen wird. Absprachen zu diesem Erstattungsverfahren erfolgen nur zwischen einem Teil der Hersteller und der Krankenkassen. Wenn zum Beispiel die Firma Novartis entsprechende Absprachen mit der GWQ ServicePlus AG, einer Gruppe mittelständischer Betriebskrankenkassen, trifft, vertritt die GWQ nur ihre Aktionäre. Die zwischen diesen Partnern getroffene Rückzahlungsvereinbarung gilt nicht für selbstzahlende Patienten sowie die nicht in der GWQ organisierten Krankenkassen. Eine solche selektive Preisabsprache für zugelassene Medikamente, bei denen ein Zusatznutzen durch den G-BA (Gemeinsamer Bundesausschuss) festgestellt wurde, kennt das deutsche Gesundheitssystem nicht ( 1 130b SGB V). Stattdessen müssen derartige Absprachen immer von allen Akteuren für alle Versicherten getroffen werden. Nur so kann das Prinzip der Chancengleichheit als grundlegende Prämisse des im Bereich der Krankenversicherung nach § 1 SGB V in Verbindung mit dem in Grundgesetz (Art. 20 Abs. (1)) normativ etablierten Solidarprinzip gewahrt werden.

\section{Kann ein am individuellen Therapieergebnis orientiertes Erstattungsverfahren ethisch problematische Anreize zur Kosteneinsparung setzen?}

Anders als die aktuelle Finanzierungspraxis der jetzigen Standardtherapie dürfte das am individuellen Therapieergebnis orientierte Erstattungsverfahren Anreize zu Kosteneinsparung setzen, die ethisch problematische Folgen nach sich ziehen. Die Behandlung mit CAR-T-Zellen ist für einen Teil der Patienten nicht alternativlos indiziert. Für Patienten mit Rezidiv eines diffusen großzelligen B-Zell-Lymphoms (DLBCL) können in der dritten Therapielinie aktuell auch andere standardisierte Therapien, nämlich eine kurative allogene Stammzelltransplantation oder eine palliative Chemotherapie erwogen werden. Bei beiden Therapieformen stehen die damit verbundenen voraussichtlichen Kosten a-priori fest und werden nicht vom individuellen Therapieergebnis abhängig gemacht. Für das Verfahren der allogenen Stammzelltransplantation ist weiterhin zu berücksichtigen, dass die Stammzellen vom Spender kostenfrei zur Verfügung gestellt werden. Die Kosten für die Transplantation in der klinischen Versorgung sind auf eine Bedarfskostenerstattung, nicht auf die Generierung eines Gewinns ausgerichtet.

Mit Einführung des am individuellen Therapieergebnis orientierten Erstattungsverfahren für eine von mehreren Therapieoptionen würde es möglich werden, mit dieser Therapie wirtschaftliche Erlöse zu erzielen, mit den Alternativen jedoch nicht. In einem z.T. marktwirtschaftlich strukturierten Gesundheitssystem könnte dies die 
Versorgungssituation dahingehend beeinflussen, dass die mit Erlös verbundene Option zukünftig begünstigt wird. Es bedarf sowohl eines medizinisch-fachlichen als auch eines normativen Diskurses, ob diese Entwicklung gewünscht wird.

Aus juristischer Sicht kollidiert das am individuellen Therapieergebnis orientierte Erstattungsverfahren mit der in Deutschland geltenden Systematik der Kostenerstattung im stationären Bereich der medizinischen Versorgung. Krankenhausbehandlungen werden in Deutschland nach $\S 85$ SGB V und § 17b Krankenhausfinanzierungsgesetz (KHG) über das Fallpauschalensystem (Diagnosis Related Groups, DRG) abgerechnet. Diesem Abrechnungssystem, das die ärztlichen Tätigkeiten und die Medikamentengabe insgesamt umfasst, ist eine vom individuellen Therapieergebnis abhängige Vergütung systemfremd. Strafrechtliche und wettbewerbsrechtliche Fragen einer solchen Einführung sind ungeklärt.

Weiterhin ist es sogar denkbar, dass durch den Preisvorteil einer erfolglosen Therapie ein sekundäres Interesse daran entstehen kann, dass die Patientin oder der Patient die Behandlung nicht überlebt (Marckmann 2015, S. $223 \mathrm{ff}$.). Ein solcher Anreiz wäre höchst problematisch.

\section{Welche Konsequenzen hat das am individuellen Therapieergebnis orientierte Erstattungsverfahren für die Selbsterhaltung der Akteure und die Generierung von Interessenkonflikten?}

Des Weiteren wird befürchtet, dass die extrem hohen Kosten der Therapie mit CART-Zellen die Selbsterhaltung aller Akteure (Krankenkassen, gesundheitliches Personal und Patient/Patientin) gefährden und beträchtliche Interessenkonflikte generieren könnten. Es sind Szenarien denkbar, in denen entweder der Patient oder die Patientin oder sogar der Arzt oder die Ärztin für die Kosten aufkommen muss, falls der Kostenträger im Nachhinein die Indikation ablehnt. Auf nur teilversicherte oder privat versicherte Patientinnen und Patienten könnten individuell sehr hohe Belastungen zukommen. Dasselbe gilt für Ärzte und Ärztinnen, die sich mit dem Vorwurf konfrontiert sehen könnten, das Präparat nicht richtig angewendet zu haben oder andere Behandlungsfehler begangen zu haben, weshalb entweder die Kosten nicht übernommen werden oder im Fall eines Versterbens des Patienten die Rückerstattung nicht gezahlt wird. Auf der anderen Seite könnte Patienten aufgrund einer monetären Risiko-Nutzen-Abwägung durch die Kostenträger die Therapie trotz realistischer Erfolgschancen vorenthalten werden. Damit wären die Zugangschancen zur CART-Zelltherapie nicht gleich und das Gerechtigkeitsprinzip wäre verletzt (Beauchamp und Childress 2013, S. 249 ff.). Ganz allgemein wird ein hohes Potenzial für Interessenkonflikte generiert, welche die betroffenen Akteure vor moralische Dilemmata stellen kann. 


\section{Werden bei der Einführung des am individuellen Therapieergebnis orientierten Erstattungsverfahrens alle relevanten ,Stakeholder“6 angemessen berücksichtigt?}

Schließlich muss gefragt werden, ob es aus ethischer Sicht angemessen ist, dass das Erstattungsverfahren ausschließlich zwischen einigen Herstellern und Krankenkassen vereinbart wurde. Sollte bei der Einführung solcher neuer und konfliktträchtiger Verfahren nicht eine größere Zahl von betroffenen Personengruppen berücksichtigt werden? Solche Betroffenengruppen bezeichnet man in der Ethik als ,,stakeholder“. Der Begriff entstammt der Wirtschaftsethik und kann im Deutschen mit „Anspruchsberechtigter“ übersetzt werden (Hansen et al. 2018). Hiermit können Personen, Institutionen, Gruppen und Organisationen gemeint sein, die einen Anspruch auf Beteiligung erheben, weil sie von einer Entscheidung primär oder sekundär, direkt oder indirekt betroffen sind (Hansen et al. 2018). Die Begründung dieses Anspruches unterscheidet sich danach, worin Betroffenheit gründet und wie sich die Beteiligung gestaltet (Hansen et al. 2018). In der von Hansen et al. vorgeschlagenen Klassifikation der Stakeholder etwa wird der normative Anspruch auf Beteiligung nach dem Grad der Betroffenheit der einzelnen Stakeholdergruppen gestaffelt. Im Rahmen dieser Klassifikation sind Patienten als Stakeholder, die objektiv und direkt betroffen sind, einzuordnen, da sie durch die Entscheidung zu einem am individuellen Therapieergebnis orientierten Erstattungsverfahren unmittelbar affiziert sind, sei es, weil damit ein monetärer Wert für das individuelle Überleben festgelegt wird, sei es, weil sie im Zweifelsfall an den Kosten beteiligt werden könnten (Hansen et al. 2018). Auch Ärztinnen und Ärzte müssen als relevante Betroffene eingestuft werden, da sie durch die Indikationsstellung und den Behandlungsvertrag haftungs- und strafrechtlich verantwortlich gemacht werden können, und zählen somit ebenfalls zu den Stakeholdern.

Dafür spricht auch, dass das Erstattungsverfahren das Arzt-Patienten-Verhältnis rechtlich beeinflussen kann. Als rechtliche Grundlage des Arzt-Patienten-Verhältnisses fungiert der Behandlungsvertrag. Der Behandlungsvertrag ist in den $\S \S 630 \mathrm{aff}$ BGB als Dienstvertrag ausgestaltet, d.h. es wird nur die Leistung an sich geschuldet, nicht das individuelle Therapieergebnis oder das Erreichen eines bestimmten Behandlungsziels. Nach dem geltenden Recht (de lege lata) wäre das Rückzahlungsversprechen des Produktherstellers ein systemwidriger Einbruch des Kaufrechts in den ärztlichen Behandlungsvertrag. Die Frage, ob medizinische Leistungen oder die Abgabe von Medikamenten analog zum Kaufrecht über das individuelle Therapieergebnis geregelt werden sollten, hat eine ethische Dimension, die sowohl Patienten als auch Ärzte betrifft. Stakeholder wie die Gruppe der Patienten oder die der Ärzte haben somit einen Anspruch, am normativen Diskurs über die Einführung und ggf. Ausgestaltung eines solchen Erstattungsverfahren beteiligt zu werden.

Zusammenfassend soll dieser Beitrag zur Diskussion darüber anregen, ob die Einführung des am individuellen Therapieergebnis orientierten Erstattungsverfahrens (P4IO) in der onkologischen Therapie vor dem Hintergrund des auf Menschenwürde sowie Chancengleichheit basierenden deutschen Gesundheitssystems ethisch vertretbar ist. Das P4IO-Verfahren bei der CAR-T-Zelltherapie hat Beispielcharakter und könnte Maßstäbe für zukünftige Vergütungsformen setzen. Eine gesellschaftliche 
Debatte über die Implementierung dieses Erstattungsverfahrens, bei der insbesondere die hier dargestellten ethischen Implikationen zu prüfen wären, sollte insbesondere unter Einbeziehung der relevanten Stakeholder geführt werden. Einige mit dem P4IO-Verfahren auftretende Probleme sind struktureller Art und können ggf. mit spezifischen Richtlinien und Handlungsanweisungen adressiert werden. Auch diese sollten jedoch vor dem Hintergrund der mit ihnen verbundenen hohen finanziellen Auflagen im Voraus thematisiert und geklärt werden.

Funding Open Access funding provided by Projekt DEAL.

Open Access Dieser Artikel wird unter der Creative Commons Namensnennung 4.0 International Lizenz veröffentlicht, welche die Nutzung, Vervielfältigung, Bearbeitung, Verbreitung und Wiedergabe in jeglichem Medium und Format erlaubt, sofern Sie den/die ursprünglichen Autor(en) und die Quelle ordnungsgemäß nennen, einen Link zur Creative Commons Lizenz beifügen und angeben, ob Änderungen vorgenommen wurden.

Die in diesem Artikel enthaltenen Bilder und sonstiges Drittmaterial unterliegen ebenfalls der genannten Creative Commons Lizenz, sofern sich aus der Abbildungslegende nichts anderes ergibt. Sofern das betreffende Material nicht unter der genannten Creative Commons Lizenz steht und die betreffende Handlung nicht nach gesetzlichen Vorschriften erlaubt ist, ist für die oben aufgeführten Weiterverwendungen des Materials die Einwilligung des jeweiligen Rechteinhabers einzuholen.

Weitere Details zur Lizenz entnehmen Sie bitte der Lizenzinformation auf http://creativecommons.org/ licenses/by/4.0/deed.de.

Interessenkonflikt J. König, C. Gerst, L. Trümper, G.G. Wulf und C. Wiesemann geben an, dass kein Interessenkonflikt besteht.

\section{Literatur}

Bach PB, Giralt SA, Saltz LB (2017) FDA approval of Tisagenlecleucel. JAMA 318:1861. https://doi.org/ 10.1001/jama.2017.15218

Beauchamp TL, Childress JF (2013) Principles of biomedical ethics, 7. Aufl. Oxford University Press, New York

Burgdorf F, Kleudgen S, Diel F (2009) Pay for Performance: Wege zur qualitätsorientierten Vergütung. Dtsch Arztebl 106:A-2190/B-1877/C-1837

Deutscher Ethikrat (2011) Nutzen und Kosten im Gesundheitswesen - Zur normativen Funktion ihrer Bewertung. Stellungnahme. Deutscher Ethikrat, Berlin

European Medicines Agency (2018) Kymriah: EPAR-product information. https://www.ema.europa.eu/ en/documents/product-information/kymriah-epar-product-information_de.pdf. Zugegriffen: 9. Nov. 2019

Gemeinsamer Bundesausschuss (2019) Beschluss des Gemeinsamen Bundesausschusses über eine Änderung der Arzneimittel-Richtlinie (AM-RL): Anlage XII - Beschlüsse über die Nutzenbewertung von Arzneimitteln mit neuen Wirkstoffen nach § 35a SGB V - Tisagenlecleucel (diffus großzelliges B-Zell-Lymphom). Gemeinsamer Bundesausschuss. https://www.g-ba.de/downloads/39-2613700/2019-03-07_AM-RL-XII_Tisagenlecleucel-DLBCL_D-375_BAnz.pdf. Zugegriffen: 09. Nov. 2019

Hansen SL, Holetzek T, Heyder C, Wiesemann C (2018) Stakeholder-Beteiligung in der klinischen Forschung; eine ethische Analyse. Ethik Med 30:289-305.https://doi.org/10.1007/s00481-018-0487-7

Korzilius H, Osterloh F, Schmedt M (2019) Interview mit Dr. med. Antje Haas, Leiterin der Abteilung Arznei- und Heilmittel beim GKV-Spitzenverband, Prof. Dr. med. Wolf-Dieter Ludwig, Vorsitzender der Arzneimittelkommission der deutschen Ärzteschaft und Dr. rer. nat. Jutta Wendel-Schrief, Market Access Director bei MSD Sharpe \& Dohm. Dtsch Arztebl 116:B203-B206

Lin JK, Muffly LS, Spinner MA, Barnes JI, Owens DK, Goldhaber-Fiebert JD (2019) Cost effectiveness of chimeric antigen receptor T-cell therapy in multiply relapsed or refractory adult large B-cell lymphoma. J Clin Oncol 37:2105-2119. https://doi.org/10.1200/JCO.18.02079 
Madlberger D (2019) Novartis Pharma GmbH und GWQ ServicePlus schließen Vertrag über ein innovatives Erstattungsmodell für die CAR-T-Zelltherapie. Novartis Pharma GmbH. https://www. finanznachrichten.de/nachrichten-2019-03/46132853-dgap-news-novartis-pharma-gmbh-und-gwqserviceplus-schliessen-vertrag-ueber-ein-innovatives-erstattungsmodell-fuer-die-car-t-zelltherapiedeutsch-016.htm. Zugegriffen: 9. Nov. 2019

Marckmann G (2015) Praxisbuch Ethik in der Medizin. Medizinisch Wissenschaftliche Verlagsgesellschaft, Berlin

Matthes N (2019) USA: Qualitätsbasierte Vergütung verbessert das Outcome. Dtsch Arztebl 116:A-248/B207/C-207

Neelapu SS, Locke FL, Go WY (2018) CAR T-cell therapy in large B-cell lymphoma. N Engl J Med 378:1065. https://doi.org/10.1056/NEJMc1800913

Quante M (2014) Menschenwürde und personale Autonomie; Demokratische Werte im Kontext der Lebenswissenschaften. Meiner, Hamburg 